\title{
Minimally invasive repair of pectus carinatum by modification of the Abramson technique
}

\author{
Muharrem Özkaya, Mehmet Bilgin \\ Thoracic Surgery Department, Antalya Health Practice and Research Center, Health Sciences University, Antalya, Turkey \\ Videosurgery Miniinv 2018; 13 (3): 383-387 \\ DOI: https://doi.org/10.5114/wiitm.2018.75888
}

\begin{abstract}
Introduction: The minimally invasive pectus carinatum surgery described by Abramson has been performed in many centers. We modified the "sub-muscular tunnel creation" part of the original Abramson technique.

Aim: To compare the operative time between the original Abramson technique and a lightly modified approach.

Material and methods: A retrospective review of 84 patients who underwent minimally invasive repair of pectus carinatum deformity between July 2008 and November 2017 was performed. We applied two different techniques, the original Abramson technique and the modified technique. Sixty-eight patients - 49 (72\%) males and 19 (28\%) females - underwent minimally invasive repair of a pectus carinatum deformity as described by Abramson (Abramson group). Sixteen patients - 13 (81\%) males and 3 (19\%) females - were operated on by our modified Abramson technique at our institution (Modified group).

Results: Eighty-four patients were included in this study: 68 patients from the Abramson group and 16 patients from the Modified group. There was no significant difference between the groups with respect to preoperative demographic features, including median age and sex. Median operative time was significantly shorter in the Modified group than the Abramson group (43 min, range: 32-54 min) in the Modified group vs. 30 min (range: 20-35 min) in the Abramson group ( $p<0.001)$.

Conclusions: This article describes a modification of a surgical technique that is safely and easily used in minimally invasive correction of pectus carinatum deformities, with minimal complications, high satisfaction rates and shorter operative time.
\end{abstract}

Key words: minimal invasive surgery, pectus carinatum, modification.

\section{Introduction}

Pectus carinatum is a protrusion of the anterior chest wall that affects $1: 1500$ births [1]. It is more common in males and typically presents during childhood and develops dramatically during pubertal growth [1]. There are two different types of pectus carinatum. One of them is the chondrogladiolar type, in which the gladiolus and inferior costal cartilage protrude along the most prominent aspect of the sternum. The other is the chondromanubrial type, in which the manubrium and superior costal cartilage protrude [2]. In contrast to pectus excavatum (funnel chest), pectus carinatum is not concealed by clothing. Patients with pectus carinatum mainly suffer from cosmetic issues, resulting in a reduced self-image and a lower quality of life compared with control patients without this deformity [3].

During the past 50 years the Ravitch open-surgery procedure [4] and its modifications, consisting of subperichondreal resection of cartilage and reconstruction of the sternum, have been the stan-

\section{Address for correspondence}

Muharrem Özkaya, Thoracic Surgery Department, Antalya Health Practice and Research Center, Health Sciences University,

07100 Antalya, Turkey, phone: +90 2422494400, fax: +90 2422494400, e-mail: drmuharremozkaya@gmail.com 
dard surgical approach for the correction of pectus carinatum. Recently, however, the minimally invasive Abramson procedure (or reversed Nuss procedure) [5], in which a steel bar is placed subcutaneously over the sternum and fixed to the ribs bilaterally, in order to restore normal thoracic shape, has gained acceptance and is also being widely performed.

\section{Aim}

We have been performing the minimally invasive Abramson procedure for the correction of pectus carinatum since 2008 [6]. In 2016, we modified the original Abramson technique and used no trocar or polyvinyl chloride (Argyle PVC tube) when moving the bar. The aim of this study is to compare the operative time between the original Abramson technique and a lightly modified approach.

\section{Material and methods}

From July 2008 to August 2017, a total of 84 patients were operated on for minimally invasive repair for pectus carinatum deformity. The first 9 patients had been previously reported [6]. A retrospective review of 84 patients who underwent minimally invasive repair of pectus carinatum deformity between July 2008 and November 2017 was performed. We applied two different techniques, the original Abramson technique and the modified technique. Between 2008 and 2016, 68 patients - 49 (72\%) males and 19 (28\%) females - underwent minimally invasive repair of a pectus carinatum deformity as described by Abramson (Abramson group). In 2016, we modified the original Abramson technique and started using it on our pectus carinatum patients. Between June 2016 and November 2017, 16 patients - 13 (81\%) males and 3 (19\%) females - underwent our modified Abramson technique at our institution (Modified group). Written consent was obtained from all patients or from their parents if their age was under 18 years. Approval for this study from the medical ethical committee was not required.

All patients presented with cosmetic complaints. A compression test was performed to check the flexibility of the chest wall, by compressing the sternum while the patient was leaning against a wall. The sternum was compressible in all patients. A detailed physical examination was performed on all patients. All patients underwent complete blood counts, routine biochemical tests, and posteroanterior and lat- eral chest X-rays. No computed tomography was obtained and also no indices were calculated. There were no abnormal findings on routine preoperative cardiac and pulmonary function evaluations. Patient satisfaction was evaluated with the answers provided on questionnaires completed on postoperative day 2. Follow-up was on postoperative days 15 and 30. The operative time was defined as minutes from skin incision to skin closure. All operations were performed by the same surgeon (M.B.).

Surgical technique for repair of the pectus defect in the Abramson group was based on the minimally invasive Abramson method [5]. According to Abramson et al. [5], the most problematic step of the procedure was subpectoral bar placement, which requires a PVC tube with a trocar. We modified this technique at this step by using two curved Doyen intestinal clamps at the same time bilaterally in order to create a submuscular tunnel so that no polyvinyl chloride tube or trocar was necessary. Surgical technique in the Modified group differs from the original technique with this minor modification.

In our previous 8-year experience performing the original procedure we observed that sharp dissection with a trocar was problematic and causing subcutaneous, intramuscular or supramuscular placement of the bar. The Doyen bowel clamp is a "blunt tipped" instrument and gives the advantage of "blunt dissection" which is more controlled than the sharp dissection with a trocar. Also it is powerful enough to penetrate the fascial layer of the pectoralis major muscle. Dissection was performed at the same time at both sides until the tips of both Doyen bowel clamps were attached. The tip of one of the Doyen bowel clamps was left open about $1-2 \mathrm{~cm}$ so as to grasp the bar (Figures 1-4). In this way it can guide and also pull the bar at the same time (the bar is transferred from one side to the other by this grab, push and pull technique).

Patient demographics, complications, and postoperative surveys were evaluated.

\section{Statistical analysis}

Statistical analysis was performed using SPSS version 18.0 for Windows (SPSS, IBM Inc., Chicago, Illinois, US) and MedCalc for Windows, version 12.2.1.0 (MedCalc Software, Ostend, Belgium). Descriptive statistics of categorical variables are given as numbers or percentages; continuous variables 


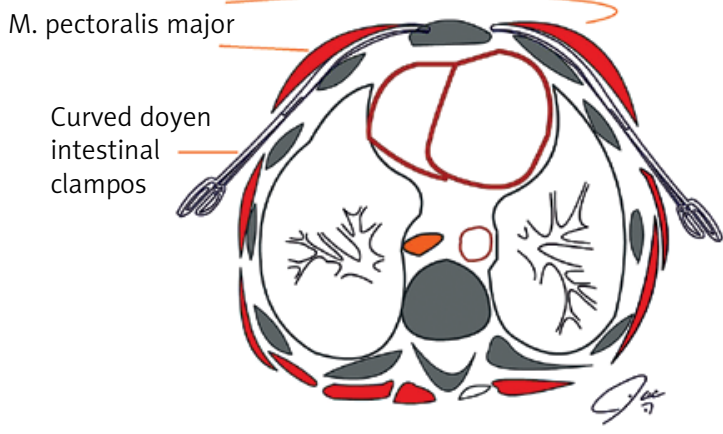

Figure 1. A tunnel is created by subpectoral dissection which is made at the same time at both sides until the tips of both Doyen bowel clamps are attached

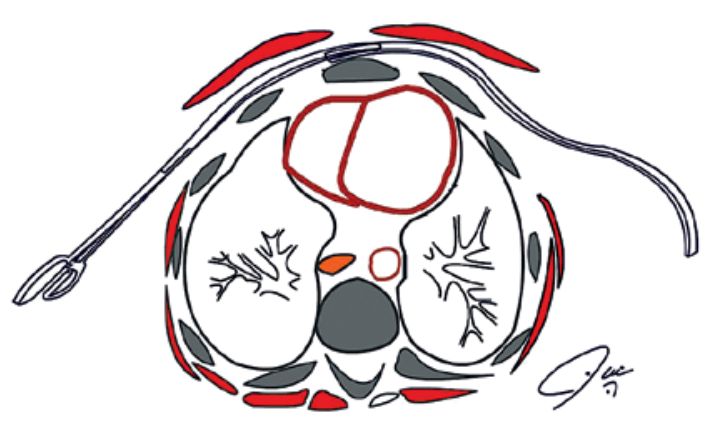

Figure 3. Grasping the bar with curved Doyen intestinal clamp

are given as medians (interquartile range). The $\chi^{2}$ test was used to evaluate categorical variables. The Mann-Whitney U-test was used to compare the medians of variables. P-values were two-sided, and values less than 0.05 were considered statistically significant.

\section{Results}

Eighty-four patients were included in this study: 68 patients from the Abramson group and $16 \mathrm{pa}$ tients from the Modified group. There was no significant difference between the groups with respect to preoperative demographic features, including median age (15 years, range: $13-16$ years) in the Abramson group and 15 years (range: $12-18$ years) in the Modified group ( $p=0.348)$ and sex (49 (72\%) patients in the Abramson group and 13 (81\%) pa-

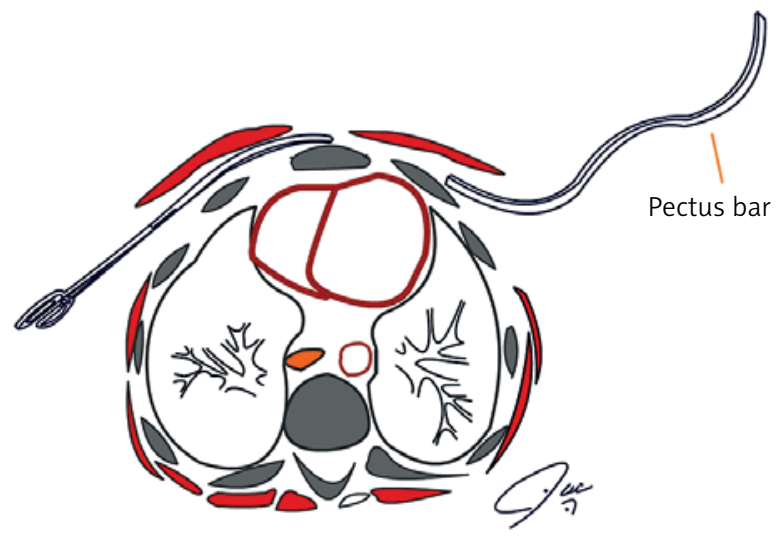

Figure 2. The bar is placed through this track and advanced into the Doyen bowel clamps whose tips are left open about $1-2 \mathrm{~cm}$ so as to grasp the bar

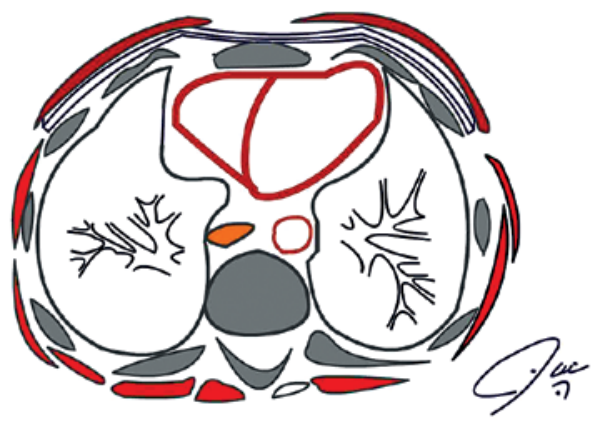

Figure 4. The tip of the bar is pulled out to the other side and fixed to the lateral thoracic wall muscles

tients in the Modified group were male; $p=0.543$ ). Demographic data, complications and duration of the operation and their distributions between the two groups are shown in Table I.

Median operative time was significantly shorter in the Modified group than the Abramson group (43 min, range: $32-54 \mathrm{~min}$ ) in the Modified group vs. 30 min (range: 20-35 min) in the Abramson group $(p<0.001)$. Comparison of the median operative times between groups is shown in Figure 5 .

In the first patient who was also the first patient of the Abramson group, the bar which was inserted subcutaneously perforated the skin on day 15 following surgery. This bar was removed and the deformity was corrected with the Ravitch method. The other patient with skin perforation was also the last patient of the Abramson group. Skin perforation in this 
Table I. Demographic data, complications and duration of operation and their distributions between groups

\begin{tabular}{|lccc|}
\hline Characteristic & Abramson $(n=68)$ & Modified $(n=16)$ & $P$-value \\
\hline Age, median (interquartile range) & $15(13-16)$ & $15(12-12.75)$ & 0.348 \\
\hline Gender, male, $n(\%)$ & $49(72)$ & $13(81)$ & 0.543 \\
\hline Complication, $n(\%)$ & $6(9)$ & $1(6)$ & 1.000 \\
\hline $\begin{array}{l}\text { Duration of operation, median } \\
\text { (interquartile range) }\end{array}$ & $43(32-54)$ & $28(20-35)$ & $<0.001$ \\
\hline
\end{tabular}

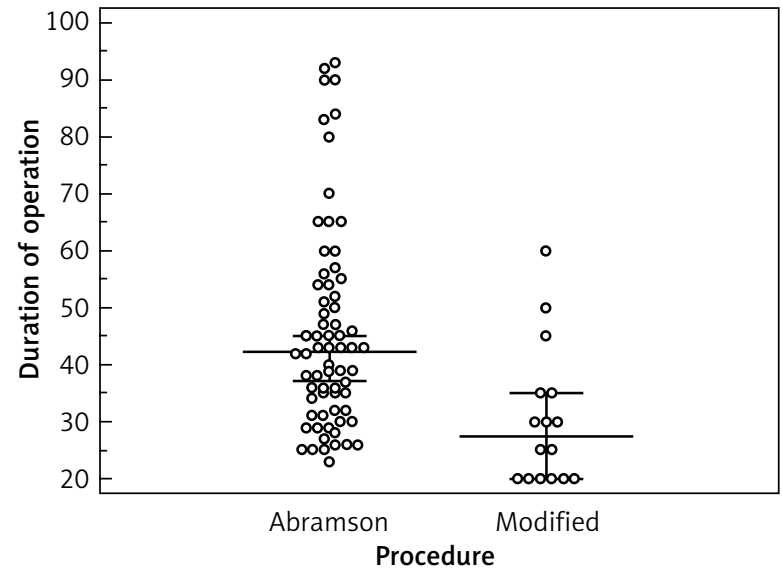

Figure 5. Comparison of the median operative times between groups

patient occurred 24 months after surgery. The bar was removed earlier than planned (at 36 months) and the skin perforation was corrected esthetically. The other complications in the Abramson group were: $1(1.4 \%)$ patient with skin hyperpigmentation, 1 (1.4\%) patient with pneumothorax, 1 (1.4\%) patient with overcorrection and 1 (1.4\%) patient with intolerable pain.

The only complication was pneumothorax in $1(6 \%)$ patient in the Modified group and no chest tubes or drains were required in any postoperative course.

No deaths or significant intraoperative morbidities occurred in either group. Blood loss was minimal and no transfusion was required. No nickel allergy was observed. No steel wire breakage or costal osteolysis was seen in the postoperative period. We had no implant failure in any of our patients at follow-up on postoperative days 15 and 30 .

Except one patient in the Abramson group (the patient with intolerable pain) all patients were satisfied with the results. Because earlier bar removal may cause recurrence of the carinatum, we aimed to keep the bar in place for at least 24 months in patients younger than 18 years and 36 months in patients older than 18 years. Since the primary operation in 2008, we removed 35 of 68 patients' implants and the average follow-up since the primary operation was 25.6 months. On the other hand, none of the implants of patients with the modified technique has been removed yet.

\section{Discussion}

For the last 50 years the standard surgical approach to pectus repair has been based on the techniques described by Ravitch [7]. The basic principles include subperichondrial removal of the costal cartilage, remodeling of the sternum, and stabilization [8]. But the skin incision made in the Ravitch procedure results in a large vertical midline chest wall scar in patients, raising esthetic concern. Furthermore, many primary care physicians view this procedure as an aggressive radical approach for such a benign condition. Thus, many patients are referred for minimally invasive techniques.

In 2005, Abramson described the technique of minimally invasive surgery for correction of pectus carinatum [9]. However, this article was published in Spanish and did not have a widespread clinical impact in the English-speaking world. Later, in 2009, an English article was published describing experience in 40 patients [5]. Adherence of the skin to the bar was the most frequent complication in the first 8 patients and the reason for this complication was the subcutaneous placement of the bar. By the subpectoral placement of the bar, however, this complication was avoided in the last 16 patients [5].

In the last 10 years, minimally invasive surgery for correction of pectus carinatum has gained worldwide acceptance among surgeons and patients $[5,6$, 10-13]. Until 2016 we used the original Abramson technique in 68 patients. As Abramson [5] men- 
tioned before, the most problematic step of the procedure was subpectoral placement of the bar. This step requires a PVC tube with a trocar. Our modification not only simplifies the most challenging part of the procedure but also eliminates the necessity of a PVC tube with a trocar. Instead of providing a PVC tube with a trocar, we developed maneuvers based on ordinary surgical instruments that are present in any thoracic instrumental surgical kit.

In 2009, Kalman [11] reported a modification to the Abramson technique in which a long curved clamp is used to enter the thoracic cavity at the mid-axillary line, and then is advanced horizontally and medially to move out through the next intercostal space. We also used a curved clamp without entering the thoracic cavity. In 2011, Perez et al. [14] published a modification with only 2 patients, in whom one end of the pectus bar was inside and the other end outside the chest. They suggested that sub-mammary and peri-areolar incisions were cosmetically more acceptable than the lateral incisions.

To our knowledge, this is the first article precisely describing meticulous dissection with a curved clamp over the sternum. This was not only a time-sparing modification but also more cost-effective (by not using a PVC tube with a trocar, operative costs were reduced by about $2 \%$ per case).

\section{Conclusions}

Our simple modification to the Abramson technique can safely and easily be used in minimally invasive correction of pectus carinatum deformities in selected patients, with minimal complications and high satisfaction rates.

\section{Conflict of interest}

The authors declare no conflict of interest.

\section{References}

1. Fonkalsrud EW. Surgical correction of pectus carinatum: lessons learned from 260 patients. J Pediatr Surg 2008; 43: 1235-43.

2. Egan JC, DuBois JJ, Morphy M, et al. Compressive orthotics in the treatment of asymmetric pectus carinatum: a preliminary report with an objective radiographic marker. J Pediatr Surg 2000; 35: 1183-6.

3. Steinmann C, Krille S, Mueller A, et al. Pectus excavatum and pectus carinatum patients suffer from lower quality of life and impaired body image: a control group comparison of psycho- logical characteristics prior to surgical correction. Eur J Cardiothorac Surg 2011; 40: 1138-45.

4. Ravitch MM. The operative correction of pectus carinatum (pigeon breast). Ann Surg 1960; 151: 705-14.

5. Abramson H, D'Agostino J, Wuscovi S. A 5-year experience with a minimally invasive technique for pectus carinatum repair. J Pediatr Surg 2009; 44: 118-23.

6. Bilgin $M$, Oral A. Early results of minimal invasive surgery in patients with pectus carinatum. Turk Gogus Kalp Dama 2012; 20: 307-11.

7. Ravitch MM. The operative treatment of pectus excavatum. Ann Surg 1949; 129: 429-44.

8. Ravitch MM. Congenital Deformities of the Chest Wall and Their Operative Correction. WB Saunders, Philadelphia 1977; 145-58.

9. Abramson H. A minimally invasive technique to repair pectus carinatum. Preliminary report [Spanish]. Arch Bronconeumol 2005; 41: 349-51.

10. Hock A. Minimal access treatment of pectus carinatum: a preliminary report. Pediatr Surg Int 2009; 25: 337-42.

11. Kalman A. Initial results with minimally invasive repair of pectus carinatum. J Thorac Cardiovasc Surg 2009; 138: 434-8.

12. Yuksel M, Bostanci K, Evman S. Minimally invasive repair of pectus carinatum using a newly designed bar and stabilizer: a single-institution experience. Eur J Cardiothorac Surg 2011; 40: 339-42.

13. Katrancioglu O, Akkas Y, Karadayi S, et al. Is the Abramson technique effective in pectus carinatum repair? Asian I Surg 2018; 41: 73-6.

14. Perez D, Cano JR, Quevedo S, et al. New minimally invasive technique for correction of pectus carinatum. Eur J Cardiothorac Surg 2011; 39: 271-3.

Received: 27.12.2017, accepted: 11.03.2018. 